\title{
PREMISE Insect Model: Integrated Population Dynamics Model for the Ex-ante Evaluation of IPM against Insect
}

\section{Pest}

\author{
Wil Hennen ${ }^{1}$ and Aude Alaphilippe ${ }^{2}$ \\ 1. Agricultural Economics Research Institute (Wageningen UR-LEI), P.O. Box 29703, 2502 LS The Hague, The Netherlands \\ 2. INRA, UERI Domaine de Gotheron, F-26320 Saint Marcel-lès-Valence, France
}

\begin{abstract}
Codling moth Cydia pomonella L. is the most serious pest of apple and pear worldwide and causes damage and decreased yields. To minimize this risk, IPM tools can be applied to reduce the use of chemicals. A cost-effective application of IPM depends on the number of insects at the time of application. Several conditions and factors influence the lifecycle and numbers of generations within a year. In order to perform ex-ante evaluations of the cost-effectiveness of IPM measures against pest insects, the generic PREMISE insect model has been developed, which integrates insect's population dynamics, the climate and other conditions, applied measures, economics, environment and human health (residues). This paper describes these components of the PREMISE insect model, and how these components interact to assess the cost-effectiveness of IPM measures and the effects on the environment and human health. Codling moth is used as a case study for this generic model.
\end{abstract}

Key words: IPM, integrated model, population dynamics, economics, environment, residues, codling moth.

\section{Introduction}

Codling moth Cydia pomonella L. is the most serious pest of apple (Malus domestica) and pear (Pyrus communis) worldwide, with up to $95 \%$ injury if the fruits are not protected [1]. A larva that feeds inside an apple will result in a rotten apple. Such damaged fruit is unsuitable for consumption and will drastically lower the orchard yield. Farmers will try to minimize the risk of crop failure by taking adequate measures to protect the crop. Application of pesticides will decrease the economic impact of the pest for the farmer, but on the other hand increase the risk for the environment and human health.

Various IPM tools are available to control this pest with less side-effect on environment and human health. There are different types of tools: (1) reduction of chemicals by timely application [2]; (2) sterile insect

Corresponding author: Wil Hennen, Ph.D., research fields: bio-economic risk models and spatial analysis. E-mail: wil.hennen@wur.nl. techniques [3]; (3) exclusion netting as an innovative tool under development [4]; (4) the application of predation and nematodes $[5,6]$; (5) application of Granulovirus and resistance management [7]. Strategies to control codling moth are described by Simon et al. [8].

However, the implementation of IPM tools is lower as expected due to their cost, efficacy or inherent risk of failure to control codling moth. A way to help the farmer change his strategy is to use a model showing the direct effect on their benefit of implementing IPM protection methods.

A cost-effective application of IPM against an insect pest depends on the numbers of insects at the time of application. Several conditions influence the lifecycle and numbers of generations within a year. The climate, especially the temperature, is a predominant condition that influences insect development and survival [9]. Indeed, in regions with a Mediterranean climate, the numbers of generations 
and reproductive capacity are much higher compared to Northern Europe. Additional conditions, like rainfall, wind, canopy placement, light proximity and the proximity of other plants, also affect for example the severity of codling moth in an apple orchard. The interaction between the life cycle of the insect (influenced by these conditions) and the application of measures is of such complex nature that an integrated population dynamics model is required to estimate ex-ante the cost-benefit of IPM options and their risk on the environment and human health. Those parameters are already integrated in population dynamic models, such as in Ref. [10], but the model described below offers to go further in the assessment.

The PREMISE insect model has been developed to integrate insect's population dynamics, environmental conditions, applied measures, economics (costs and yields), environment and human health (residues) in order to perform ex-ante evaluations of the cost-effectiveness of IPM tools by farmers and advisors. PREMISE is the acronym of pest risk evaluation model by integrating stage effects. The goal of this paper was to describe the PREMISE insect model and its integrated components and to illustrate that a holistic ex-ante evaluation of IPM strategies against insect pest is possible with an integrated model. Codling moth is the insect used throughout the paper to illustrate the PREMISE insect model.

A general view of the model functioning is given in section 2 and the various components (i.e., modules) are described more in detail in section 3. The test and validation of the model is presented in section 4 . Finally, some aspects of the model and its use are discussed in the last section.

\section{Model Outline}

A schematic outline of the PREMISE insect model is shown in Fig. 1. In section 3, the elements are explained and described in more detail. The population dynamics module, which is the core of this model

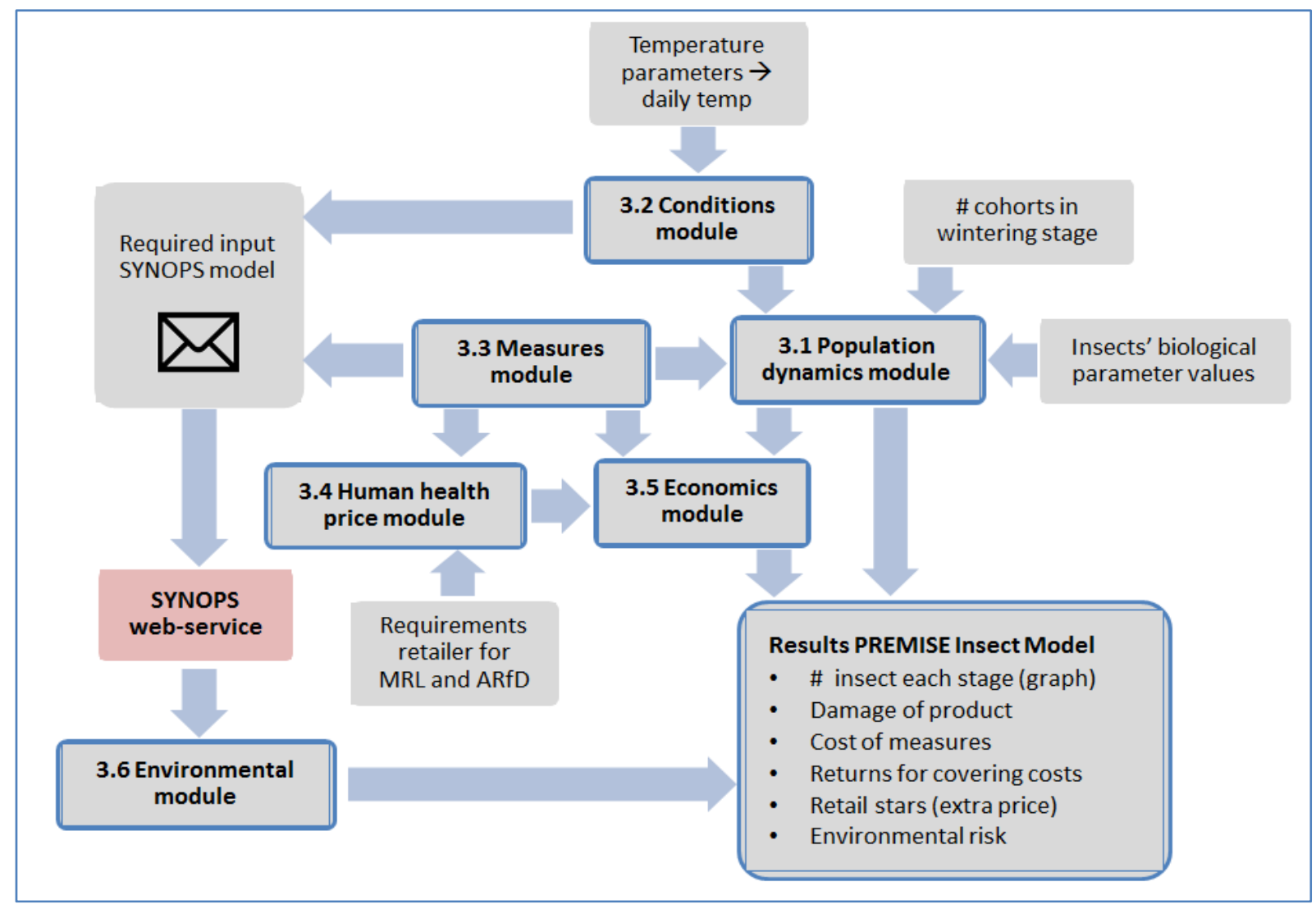

Fig. 1 Outline for PREMISE insect model (see text for explanation of modules). 
(section 3.1), uses temperature parameters, biological parameters and a parameter for the remaining cohorts from over-winter of previous year. Other conditions and circumstances also influence the dynamics. The condition module is described in section 3.2. Measures against the pest (section 3.3) influence the population unfavourably and thereby decrease the damage. Measures come at a cost and are calculated in the economics module (section 3.5). These measures, in particular chemicals, may have undesirable effects on human health because of remaining residues. The human health pricing module in section 3.4 calculates the effects, especially possible price increases (economic benefits) when complying with retailer's standards. Measures can also have undesirable environmental effect (section 3.6). PREMISE sends a request by web to the environmental risk model for the calculation of risks based on parcel specific data and the applied pesticides by the farmer.

Intended users of the model are farmers (together with their advisor) and experts.

\section{Integrated Modules of PREMISE}

The modules of the PREMISE insect model are described more in detail in this section. In Fig. 1, a general overview and the way that these modules are related are shown.

\subsection{Population Dynamics Module}

Taking the paper in Ref. [9] as a starting point, a population dynamics module based on iterative-cohort technique has been developed from scratch. This population dynamics module is imperative for the model. In a model that was developed for apple scab (PREMISE apple scab model) [11], the population dynamics are simulated with rather simple arithmetical formulas. Contrary to this scab model, the PREMISE insect model has a solid base with the model components as described by Shaffer and Gold [9].

PREMISE distinguishes six stages for the population dynamics module (Fig. 2). It starts with the overwintering cohorts, which are influenced by sanitation measures in the previous year. The user can assign the number of cohorts. From the start of the year and when the temperatures are rising, the numbers of adults increase and start mating. The females produce eggs, and after hatching, the organism is in a "searching" stage before entering an apple. In the apple, it becomes a larvae, followed by the stages of pupae and adult. Depending on the temperature, successive generations within one year can appear (up to three) [12].

Parameter values for the population dynamics are entered by the expert. For each stage, there are parameters for the calculation of the rate of development, the production and the loss. In Ref. [9], there is detailed information on the arithmetic formulas and parameters. This information is used for the development of the module. On a daily basis, the number of individuals within each stage can be estimated by the formulas. Fig. 3 gives an illustration of the graphical result, and the period is from early April to the end of the year.

A higher temperature will result in more individuals and more generations because of an influence on the rate of development and production. Other conditions (section 3.2) and measures (section 3.3) also influence the curves.

\subsection{Conditions Module}

The daily temperature is defined by a few parameters

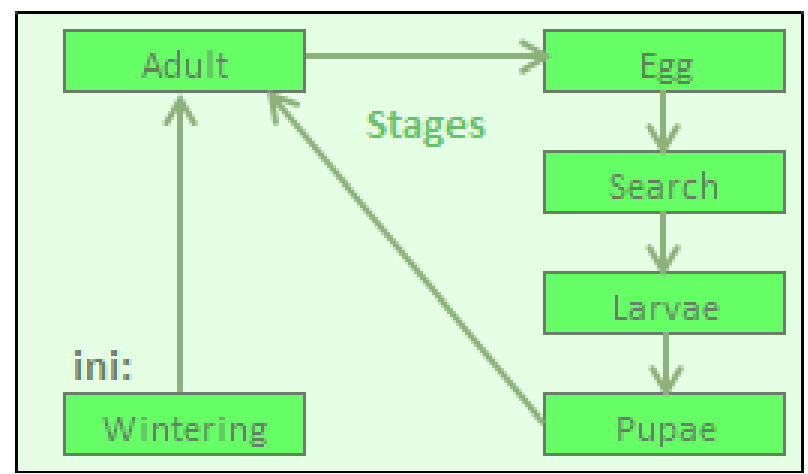

Fig. 2 Stages of insect development. 


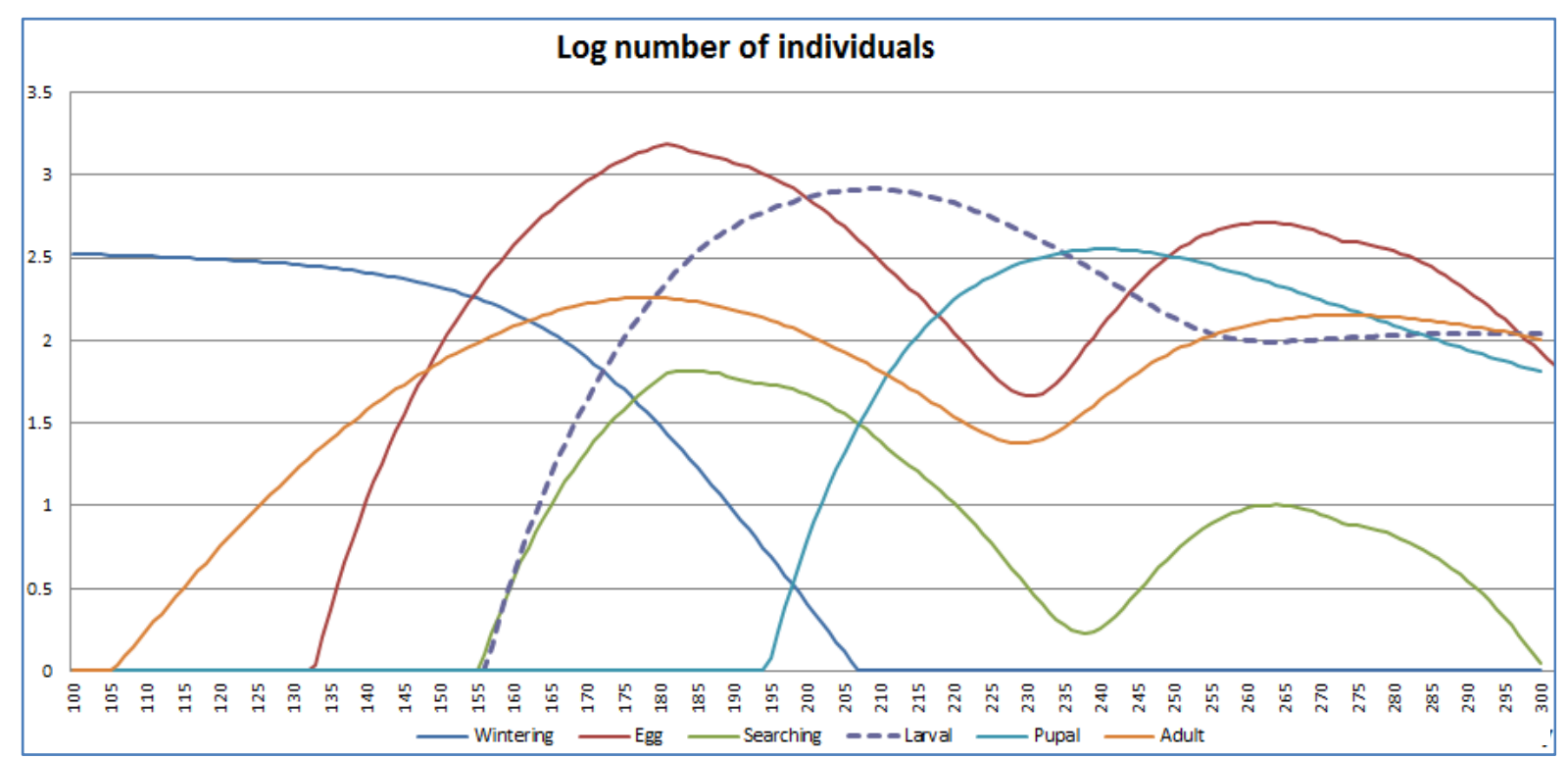

Fig. 3 Log number of individuals for each development stage during the year subject to Dutch climate conditions. Two generations can be distinguished (screen dump PREMISE model).

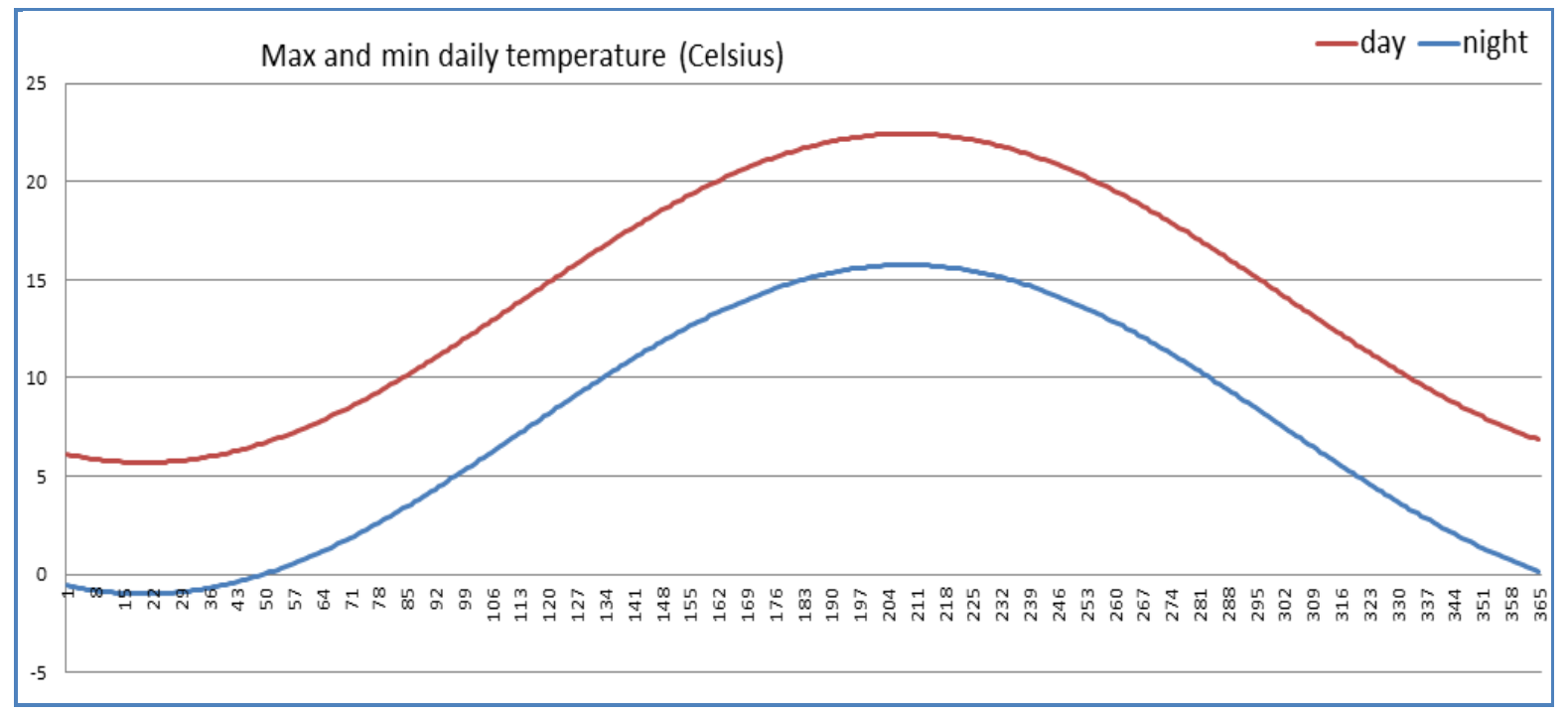

Fig. 4 Calculated temperature curve for the Dutch region based on four parameters.

and is a very important condition for the population dynamics and number of generations within a year. With four parameters, the model can calculate the daily minimum (night) and maximum (day) temperature for the whole year. These parameters are:

(1) Yearly average temperature for the region;

(2) Difference between the maximum and minimum temperature on a weekly base;

(3) The date when the temperature curve has maximum value (e.g., July 25);
(4) Difference between average night and day temperature.

With these parameter values for the Dutch region, the graphs in Fig. 4 appear. Data from these graphs are used in the conditions module.

Other site-specific conditions also influence the population dynamics, although less dominant than temperature. These conditions are rainfall, wind, regularity of canopy, trunk protection, early harvesting of the cultivar, light proximity and the proximity of 
other plants. These conditions affect the severity of the insect. The user can assign whether a condition is favourable, unfavourable or absent for the development of the pest. For example, if there is much rainfall in the area, then this is an important cause of mortality among larvae and therefore unfavourable for the insect.

\subsection{Measures Module}

With the IPM measures module, the user can define and select the measures, the period of their application (by start-day and end-day), the stage that is affected (e.g., eggs when an egg treatment is selected), the effect on the population of that specific stage and the product price of one application.

The user is not restricted by the model to enter the type of application, i.e., the name (e.g., "Carpovirusine"), the model only requires values for the parameters.

Sanitation measures like removing remaining fruits after harvest can be applied. This will influence the level of infestation as start for the next year to come. In the model, especially the chemicals play a key role. They influence the population dynamics, the economics, the environment and human health.

\subsection{Human Health Pricing Module}

The application of chemicals as measures against the pest may result in undesirable residues on the harvested product which can be harmful for human health [13]. The type and amount of chemicals and the time between application and harvest influence the amount of residue.

The first-order kinetic equation from Ref. [14] is used for the calculation of the residue that remains on the product. The amount expressed in ppm (or $\mathrm{mg} / \mathrm{kg}$ ) is calculated from the active ingredients, the period for DT50 (lethal dose which will kill $50 \%$ of the population), number of days before harvest, the fraction of spray intercepted, surface area of the commodity and the crop leaf surface area.

If the residue level of the fruit complies with the requirements of a wide range of supermarkets, then a price premium is possible. The requirements are additional to the maximum residue limit (MRL) levels demanded by law. A supermarket may impose additional restrictions on the number of different chemicals applied, on the MRL of an individual chemical, on the sum of MRLs, on the acute reference dose (ARfD) of an individual chemical and on the sum of ARfDs. In the model, the number of stars (or price premiums) and thus the price increase (\%) depend on the percent of retailers that will accept. All inputs (requirements, price increase, percent of retailers) are supplied by the expert and reflect the situation in the specific region or country and the type of fruit (apples). If the fruit is sold by supermarkets in other countries (export), then the requirements of these supermarkets should also be taken into account.

The relation between meeting retail standards and expected additional price is not obvious at the moment, therefore assumptions of expected additional prices should be used. In the model, price increases were assumed to be $5 \%, 10 \%$ and $20 \%$, respectively, when $60 \%, 80 \%$ and $100 \%$ of the supermarkets accept.

\subsection{Economics Module}

The economic module calculates the costs (chemicals, labour, equipment linked to codling moth management) and returns based on yield, price and price reduction. Only costs that are related to the pest are considered (method of partial budgeting), and thus limit the number of economic data and parameters to collect. For each type of measure or application, the required labour (hours and wage per skill-level), equipment cost and additional fuel cost are placed in a table with region or country specific values.

Damage of fruit reduces the quality of the fruit and increases the cost for sorting. Adequate measures against the pest will decrease the infection and thus increase the yield, but residues might have a negative 
price effect. Different ex-ante IPM options can economically be compared by the model. The economic evaluation criterion is "returns for covering other costs", calculated as Eq. (1):

Returns $=$ the yield (Euros) - the costs of measures - additional sorting costs

\subsection{Environmental Risk Module}

For the assessment of environmental risk, PREMISE makes use of a connection with the web-based SYNOPS model from Julius Kühn Institute (JKI) in Germany [15, 16]. SYNOPS calculates environmental risk potentials of applied herbicides, fungicides and insecticides to protect the crops. The model distinguishes indices for aquatic (algae, daphnia, lemna, fish, chironomus), terrestrial (earthworm, bee) and groundwater risks. The geographic information system (GIS) based version of SYNOPS uses integrated regional specific information of the production area, like soil type, $\mathrm{pH}$, surface water, slope, monthly temperature and precipitation. The kind of crop (e.g., apples) and some production data are also used as input for the model (e.g., harvest date, buffer zone). Data for pesticide applications is supplied as input; for each application, it consists of the product name, amount of active ingredients, the day of application and the mode of application (e.g., drift reduction).

A web service is used as an interface between PREMISE and SYNOPS. In a web service, there is exchange of data over the web between the service requester (PREMISE) and the service provider (SYNOPS). In PREMISE, the user enters parcel specific data and data of all applied pesticides. These data are automatically wrapped in a suitable format (so-called "SOAP-protocol") and send to the service provider. SYNOPS uses these data as input for the model and sends the result instantaneously to PREMISE. After arrival, the result is automatically transformed to a table of environmental risks (Fig. 5). The whole procedure takes only $1 \mathrm{~s}$.
PREMISE and the web service request to SYNOPS are developed in visual basic for applications (VBA) with microsoft excel as user interface.

\subsection{Output PREMISE Insect Model}

Results of PREMISE can be summarized in following aspects:

Biological: number of organisms in each stage, daily as well as year total;

Economic: returns, yield damage, costs and the indicator "returns for covering costs";

Environmental: chronic and acute risks of environmental indices (reply of SYNOPS request);

Human health: comply to MRL and ARfD standards of retailers.

Not all aspects are integrated in the economic module. PREMISE does not economically valuate the environmental risk nor aggregates both aspects in another way.

\section{Model Validation}

The outcome of the population dynamics module of PREMISE was validated with the graphical outcome described in Ref. [9]. The resemblance with these figures was based on their arithmetic formulas and parameters, and the model's reaction of changes in temperature and other biological parameters were satisfying enough to assume a good working of this module.

The temperature is a dominant factor of the population dynamics. With all biological parameters set to codling moth Cydia pomonella L., the regional effects are clear when looking at the dynamics of Dutch, Southern Scandinavian and Southern France climate conditions (Figs. 4, 6a and 6b, respectively). The number of generation increases with temperature, from one (Fig. 6a) to two (Fig. 4) and to nearly three (Fig. 6b). It is noted that the development starts earlier and ends later when temperatures are higher and thus lead to an increase of the number of generations. Also the number of organisms increases with temperature, 
PREMISE Insect Model: Integrated Population Dynamics Model for the

\begin{tabular}{|c|c|c|c|c|c|c|c|c|c|c|}
\hline \multirow{3}{*}{$\begin{array}{r}\text { chronic } \\
\text { acute }\end{array}$} & Aquatic & Algae & Daphnia & Lemna & Fish & Chironomus & Terrestrial & Earthworm & Bee & Groundwater \\
\hline & 0.2994 & 0.0512 & 0.2248 & 0.0028 & 0.2994 & 0.0000 & 0.0355 & 0.0158 & 0.0355 & 2.8480 \\
\hline & 0.2946 & 0.0715 & 0.2450 & 0.0059 & 0.2946 & 0.0001 & 0.0093 & 0.0011 & 0.0093 & 14.2402 \\
\hline
\end{tabular}

Fig. 5 Transformed results from SYNOPS after request with a web service (screen dump PREMISE).

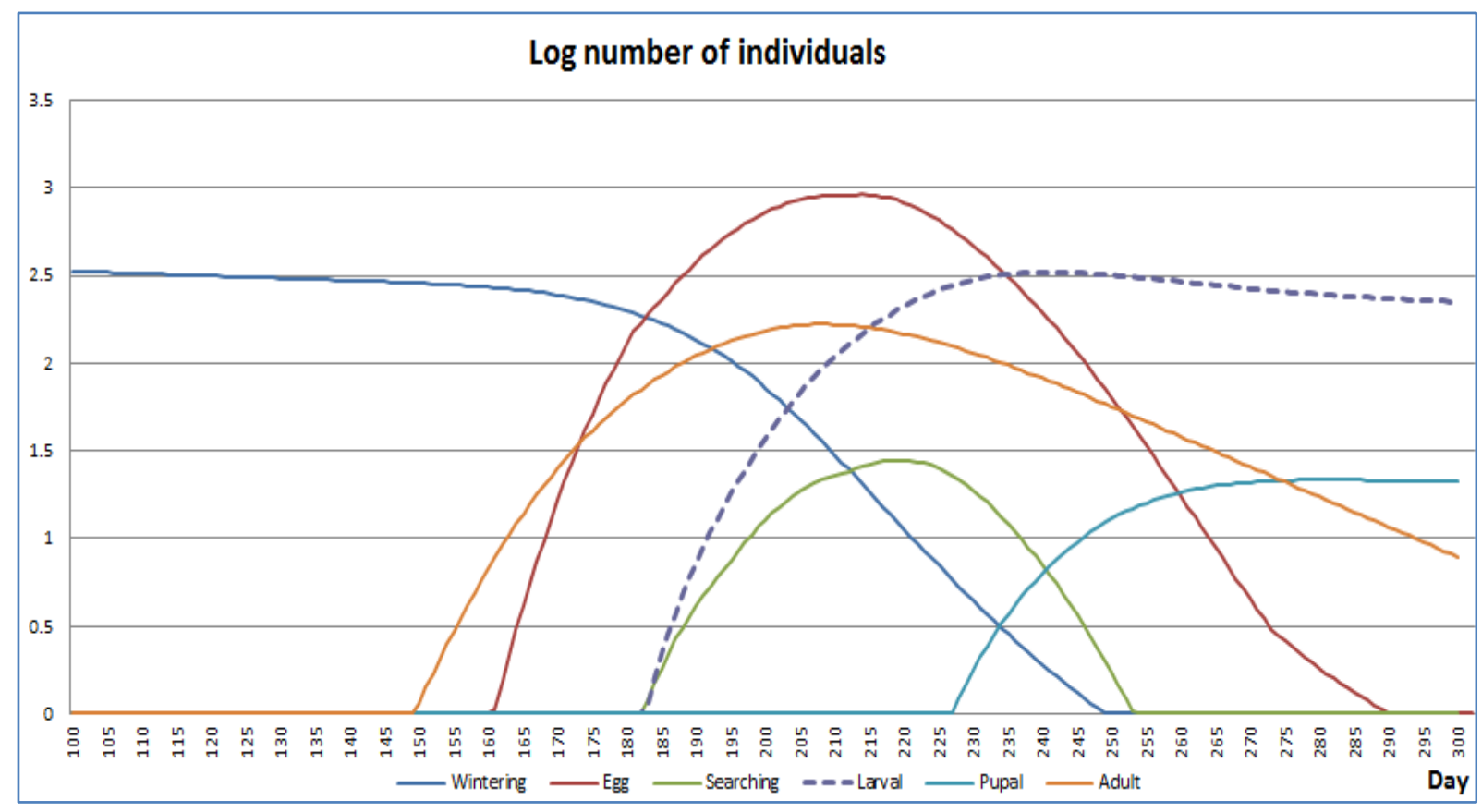

(a) Southern Scandinavia

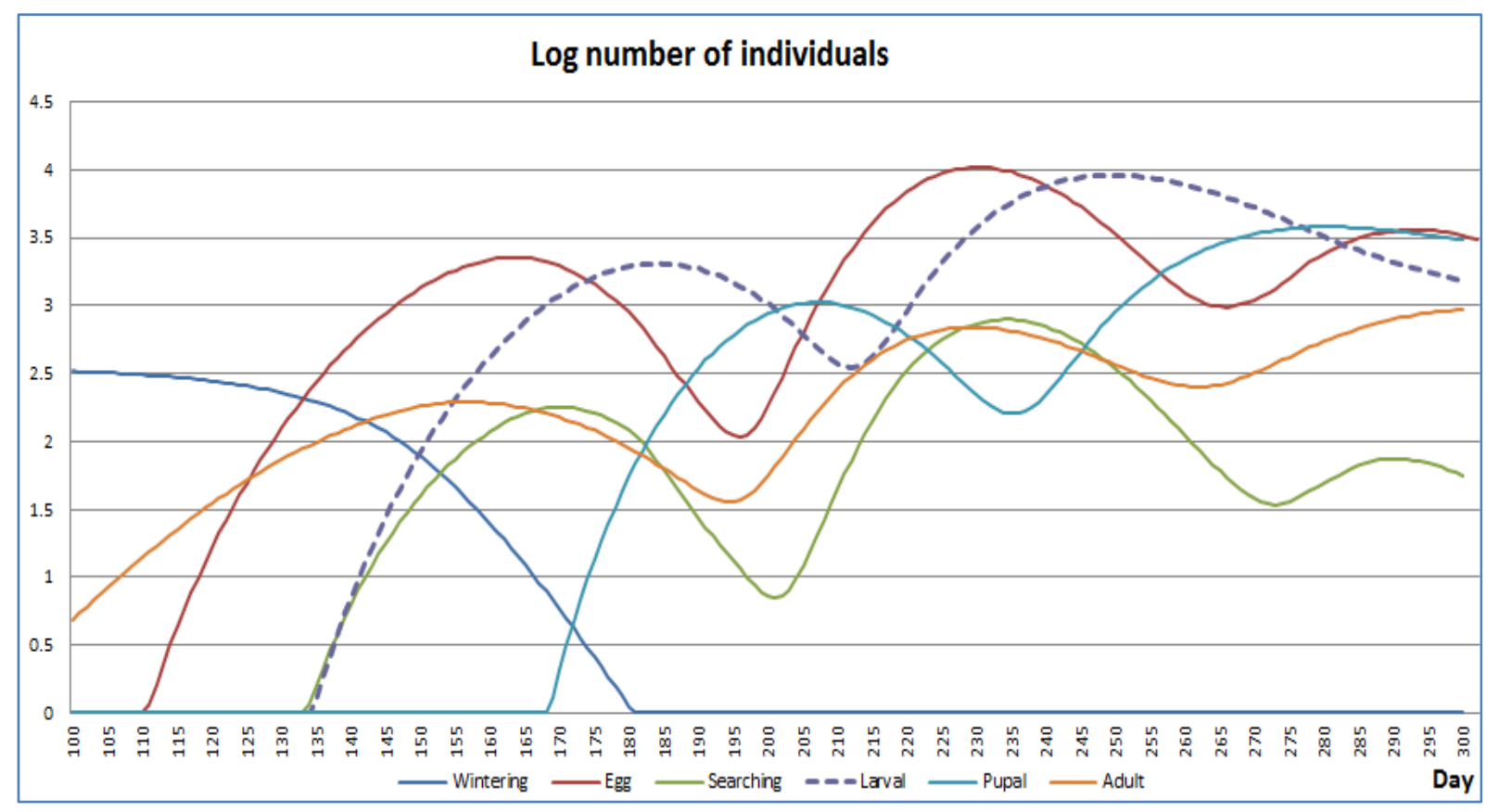

(b) Southern France

Fig. 6 Calculated temperature curve for Southern Scandinavia (a) and Southern France (b) based on four parameters. 


\begin{tabular}{|ccllc|}
\hline current run & previous run & & produced number \\
1080 & 15180 & Total \# of larvae & adult $\downarrow$ & 576 \\
4074 & 59764 & Total \# of eggs & eggs $\downarrow$ & 4074 \\
$€ 20,187$ & $€ 19,363$ & Returns per ha & search $\downarrow$ & 2176 \\
$€ 284$ & $€ 0$ & Costs measures \& add.sort & larvae $\downarrow$ & 1080 \\
$€ 19,903$ & $€ 19,363$ & Returns for covering other costs & pupae & 568
\end{tabular}

Fig. 7 Summarization of the results of PREMISE run (screen dump).

however, the log number presentation of the figures might suggest less dramatic increase. The model can also be applied for another insect, just by adapting the parameters and validating the graphical result.

Field data from an experimental orchard design (Gotheron experimental unit in France, season 2013), which has been described earlier by Simon et al. [17], were used to validate a prototype of the model. For the case study (Cydia pomonella L. in an apple orchard), the field and model results are in the same range of values. The environmental module, i.e., the web based connection with SYNOPS, was not realized in that version of PREMISE. The environmental outcome of SYNOPS has been tested earlier, and results are presented in Ref. [18].

Options can be compared with the model, e.g., different sets of measures or changed assumptions of the conditions. Fig. 7 is a screen dump of the model with the number of larvae and eggs for the two options (option "current run" and option "previous run"), the returns/ha in Euro, the costs for measures against codling moth, the additional sorting costs due to damaged fruit from codling moth, the returns for covering other costs (returns/ha - costs measures additional costs). Options with higher returns for covering costs are more preferable.

\section{Discussion}

Temperature has a dominant effect on the population dynamics, as shown in Figs. 4, 6a and 6b, and thereby the choice of IPM tools. Because of these temperature differences, an application can be cost-effective in one region but not in another region.
For example, nets might be cost-effective in Southern Europe, but they are too expensive (for investment as well as labour costs) to apply in Northern Europe. This illustrates that the type of the application and the number and periods to apply during the year are site-specific. However, this is a partial conclusion when focusing on codling moth damage alone. If other elements, such as hail risk, are included, this might give other conclusions, even for areas where codling moth pressure is not that important as in South France.

The human health pricing module still is in an infancy stage in PREMISE and requires further development, since the relation between meeting retail standards and expected additional price is not obvious at the moment and is furthermore region/ country-specific as well as retailer-specific. Lower residues will lead to increasing the number of retailers who are willing to accept the product and therefore more chances to sell at top-segments in the market with higher prices. This procedure in PREMISE coincides with the remark in Ref. [18], which suggested "a reduction of fungicide sprays might decrease the residue level of fungicides in the product, which might result in access to higher market segment and thus somewhat higher selling prices". For example, for top-quality tomatoes, the top-segment pays $20 \%-30 \%$ higher prices [19]. But a higher price is not an isolated contribution of lower level of residues. Also and above all, taste, flavour and physical quality (i.e., type/cultivar, shelf life, colour, packaging) are targets for differences in prices [19]. Product pricing is a complex integration of a number of aspects, in which human health is only one aspect to consider. Additional prices in PREMISE 
are solely assumptions.

PREMISE makes use of another model by web service for the assessment of the environmental risk. This is the web based SYNOPS model developed by JKI in Germany $[15,16]$. In this way, PREMISE makes use of a sophisticated model developed by specialists in the area of environmental risk assessment. PREMISE developers saved development time for a "comparable" module and they were restrained to make a module, for which they lacked knowledge and data. Connecting with models and databases from external sources via web services makes it possible to make use of the best available sources in the world, where specialists maintain these sources. An example to consider would be the development of a database with economic parameters for labour, equipment and other costs for a large number of countries. Each model making use of such model has a uniform and agreed upon economic value for a parameter (e.g., the wage per hour for a skilled worker on a farm in the Netherlands). Subsidies from European Union (EU) could stimulate the development and use of models and databases by research organizations. The web service between PREMISE and SYNOPS realized in the EU "pesticide use-and-risk reduction (PURE)" project is an illustration of the value of such proposal [11].

The current version of the PREMISE insect model still requires further testing with other years and in other countries with other climatic conditions. The human health pricing aspect was not validated, because the aspect of pricing related to residues and market segments need to become more clear in future. The PREMISE insect model is not limited to the case study Cydia pomonella L.; with other biological parameters, another insect can be mimetic by the model. In this way, PREMISE should be considered as a generic model for various pests and hosts. A combination with another type of PREMISE model (on scab), developed within the EU PURE project [11], may offer perspectives for a more complete strategic cost-benefit model.

\section{Conclusions}

Modelling the cost-benefit effect of IPM measures requires interaction with population dynamics of the pest. A model that does not include population dynamics lacks a good comparison with respect to damage and is not able to show the magnitude of the expected infestation and the best time within the growing season to apply chemicals or other measures. On the other hand, a population dynamics model without economic, environmental and human health pricing aspects is not able to show economic consequences and risks when applying measures to reduce the infestation level. PREMISE has demonstrated that it is possible to integrate biological, economic, environmental and human health pricing aspects, and thus has a holistic view of the consequences of IPM measures. The web service connection with the SYNOPS model has proven the promising perspectives that such interaction can also be with other models on other locations.

\section{Acknowledgments}

The research leading to these results has received funding from the EU seventh framework programme (FP7/2007-2013) under the grant agreement No. 265865. The authors appreciate the help of Jörn Strassemeyer and Barbara van Hout for realizing the web service between PREMISE and SYNOPS, and Sylvaine Simon, Bart Heijne and Jan Buurma for reviewing PREMISE.

\section{References}

[1] Gharekhani, G. 2009. "Modeling Population Dynamics and Dispersion of Codling Moth Cydia pomonella L. (Lepidoptera, Tortricidae)." Ph.D. thesis, Faculty of Agricultural Sciences at the University of Hohenheim, Stuttgart, Germany.

[2] Brunner, J. F., Hoyt, S. C., and Wright, M. A. 1987. Codling Moth Control-A New Tool for Timing Sprays. Washington, USA: Washington State University.

[3] Vreysen, M. J. B., Carpenter, J. E., and Marec, F. 2010. 
"Improvement of the Sterile Insect Technique for Codling Moth Cydia pomonella (Linnaeus) (Lepidoptera: Tortricidae) to Facilitate Expansion of Field Application." Journal of Applied Entomology 134 (3): 165-81.

[4] Sauphanor, B., Severac, G., Maugin, S., Toubon, J. F., and Capowiez, Y. 2012. "Exclusion Netting May Alter Reproduction of the Codling Moth (Cydia pomonella) and Prevent Associated Fruit Damage to Apple Orchards." Entomol. Exper. Appl. 145 (2): 134-42.

[5] Monteiro, L. B., Lavigne, C., Ricci, B., Franck, P., Toubon, J. F., and Sauphanor, B. 2013. "Predation of Codling Moth Eggs Is Affected by Pest Management Practices at Orchard and Landscape Levels." Agriculture Ecosystems and Environment 166: 86-93.

[6] Lacey, L. A., Arthurs, S. P., Unruh, T. R., Headrick, H., and Fritts, R. 2006. "Entomopathogenic Nematodes for Control of Codling Moth (Lepidoptera: Tortricidae) in Apple and Pear Orchards: Effect of Nematode Species and Seasonal Temperatures, Adjuvants, Application Equipment and Post-Application Irrigation.” Biological Control 37: 214-23.

[7] Jehle, J. A., Eberle, K. E., Asser-Kaiser, S., Schulze-Bopp, S., and Schmitt, A. 2010. "Resistance of Codling Moth against Cydia pomonella Granulovirus (CpGV): State of Knowledge." In Proceedings of the 14th International Conference on Cultivation Technique and Phytopathological Problems in Organic Fruit Growing, 133-6.

[8] Simon, S., Defrance, H., and Sauphanor, B. 2007. "Effect of Codling Moth Management on Orchard Arthropods." Agriculture Ecosystems and Environment 122 (3): 340-8.

[9] Shaffer, P. L., and Gold, H. J. 1985. “A Simulation Model of Population Dynamics of the Codling Moth Cydia pomonella." Ecological Modelling 30: 247-74.

[10] Inoki. 2015. "Tools on Crop Protection." Accessed May 30, 2015. http://www.ctifl.fr/inoki/Pages/Presentation/ /Modele.aspx?id=2. (in French)

[11] Heijne, B., Hennen, W., and Buurma, J. 2013. "PREMISE: A Prototype of An Empirical Model of the Epidemiology of Apple Scab for Economic Assessment of IPM Tools." In Proceedings of the 8th International Conference on Integrated Fruit Production at Kusadasi
(Turkey), 229-34.

[12] Alston, D., Murray, M., and Reding, M. 2010. "Codling Moth (Cydia pomonella)." Utah Pests Fact Sheets, Utah State University Extension and Utah Plant Pest Diagnostic Laboratory. Accessed September 15, 2015. http://extension.usu.edu/files/publications/factsheet/codli ng-moths06.pdf.

[13] Gill, H. K., and Garg, H. 2014. "Pesticide: Environmental Impacts and Management Strategies." In Pesticides-Toxic Effects, edited by Solenski, S., and Larramenday, M. L. Rijeka, Croatia: Intech, 187-230.

[14] Maclachlan, D. J., and Hamilton, D. 2010. "Estimation Methods for Maximum Residue Limits for Pesticides." Regulatory Toxicology and Pharmacology 58 (2): 208-18.

[15] Strassemeyer, J., and Gutsche, V. 2010. The Approach of the German Pesticide Risk Indicator SYNOPS in Frame of the National Action Plan for Sustainable Use of Pesticides. Organization for Economic Cooperation and Development (OECD) Report 2010.

[16] Gutsche, V., and Rossberg, D. 1997. "SYNOPS 1.1: A Model to Assess and to Compare the Environmental Risk Potential of Active Ingredients in Plant Protection Products." Agriculture, Ecosystems and Environment 64 (2): 181-8.

[17] Simon, S., Brun, L., Guinaudeau, J., and Sauphanor, B. 2011. "Pesticide Use in Current and Innovative Apple Orchard Systems." Agronomy for Sustainable Development 31 (3): 541-55.

[18] Heijne, B., Helsen, H., Buurma, J., Strassemeyer, J., Holb, I., Alaphilippe, A., Simon, S., Capowiez, Y., Rossi, V., and Caffi, T. 2014. "Report on the Effects Ex-post Assessment of Selected IPM Tools and Solutions from on Station and on Farm Experiments under Well Controlled Conditions." Deliverable D5.2, PURE Project, FP7-265865. Accessed September 15, 2015. http://www.pure-ipm.eu/sites/default/files/content/files/D 5.2_vFinal.pdf.

[19] Buurma, J. 2015. "New Challenges for IPM Research in Horticulture." IPM Innovation in Europe, Institute of Plant Protection, National Research Institute and EU PURE Project, Poznań, Poland. Accessed September 15, 2015. http://www.pure-ipm.eu/node/528. 